\title{
The Rotorcraft Aerodynamics Library: A Modelica Library for Simulation of Rotorcraft Aerodynamics and Whirl Flutter
}

\author{
Cory Rupp Nicolas Reveles \\ ATA Engineering, Inc., USA, \{cory.rupp, nicolas.reveles\}@ata-e.com
}

\begin{abstract}
Rotor aeromechanics is a multidisciplinary branch in the field of rotorcraft that involves performance, loads, vibrations, stability, and noise. Aeromechanic analysis in this field is a complex task typically requiring specialized software tools to simultaneously resolve the coupled structural, mechanical, and aerodynamic solutions that contain motion (both rigid and elastic) and forces. One drawback of these tools is that they are often overspecialized and do not lend themselves well to the analysis of the latest rotorcraft advances and concepts, including those utilizing distributed electric propulsion. In this paper, a Modelica library is presented that provides an extensible platform for performing analysis and design studies of current and future rotorcraft. Several examples are presented that illustrate how the library can be used to perform aerodynamic and whirl flutter stability analysis as well as control system design for multi-rotor aircraft.
\end{abstract}

Keywords: $\quad$ Modelica, rotorcraft, aeromechanics, aerodynamics, whirl flutter, flight control

\section{Introduction}

Analytic methods for analyzing rotors are frequently capable of adequately characterizing the basic aerodynamics of rotor blades but have shortcomings in addressing more-complex configurations, as such methods are not easily extendable to adequately describe complex geometry, elastic blades, or physics such as time-resolved rotor wakes. In addition, detailed hub mechanics are often missing that can significantly affect rotor behavior and stability. Therefore, numerical methods are frequently preferred for analyzing realworld designs. Several comprehensive rotor analysis tools exist (e.g., CAMRAD-II, RCAS) with varying capabilities intended to overcome the limitations of analytical methods.

Whirl flutter is a phenomenon that principally affects propeller and proprotor aircraft that encounter high rotor inflow velocities and involves the tip path plane precessing ("whirling") in the form of a dynamic instability. The precession stems from the gyroscopic motion of the rotating blades. This otherwise stable motion is destabilized under certain conditions by aerodynamic forces introduced by changes in the local blade inflow angles due to the precession (Reed, 1967). Analytic methods exist, such as the Houbolt-Reed method (Houbolt and Reed, 1962; Reed and Bland, 1961), that are capable of quantifying stability; however, they are limited by the same factors since general rotor analysis and software is typically used once a basic rotor design is established to capture additional aerodynamic or mechanical effects.

Existing software tools for performing rotor aerodynamics and whirl-flutter analyses are generally tailored specifically to rotor analysis and therefore are limited, whether by the available connections to external tools, the rotor control schemes, or the ability to model effects such as electromechanical interaction for rotors driven by electric motors. Emerging rotorcraft designs, however, are increasingly moving toward multi-rotor and electrified rotor concepts for which these tools typically lack capability to analyze. An example is the NASA X-57 distributed electric propulsion aircraft, which consists of 14 rotors distributed along the wings intended to enhance performance under certain flight conditions. Modelica has previously been used to analyze this complex multi-physics problem (Batteh et al., 2018); however, explicit rotor dynamics and stability were not considered. Existing rotor analysis tools are not designed to handle the coupled multi-rotor aero-electrical-mechanical problem, so alternative analysis approaches are necessary.

This paper presents a new Modelica library, the Rotorcraft Aerodynamics Library (RotorAeroLib), that provides a rotor aerodynamics modeling capability and rotor mechanical templates that can be used to analyze various rotor configurations such as those found on helicopters as well as fixed wing, tilt-rotor, and distributed electric propulsion aircraft. By leveraging the capabilities and extensibility of Modelica, this library will enable engineers to perform sophisticated analyses beyond what is capable in existing comprehensive rotorcraft tools.

\section{The Rotorcraft Aerodynamics Library}

The Rotorcraft Aerodynamics Library mixes new modeling capabilities of rotor blade aerodynamics with existing Modelica Standard Library models to provide a 
toolset for coupled aerodynamic analysis and design. The rotor hub topologies used in rotorcraft vary significantly, so it is imperative to have a flexible and extensible modeling technology such as Modelica. The goal of the library is to make building aerodynamic models of rotors easier by providing a set of common building blocks.

The Rotorcraft Aerodynamics Library is organized into aerodynamic models and template implementations of rotor components, including, for example, the rotor blade as well as several example models. The top-level view of the library is shown in Figure 1. The most relevant of the models will be described in the following sections. Some of the template rotor models, e.g., the RotorBlade model, make use of the freely available Modelica Deployable Structures Library (Rupp and Schweizer, 2018) (available via the Modalica Association website), which contains an appropriate flexible beam formulation that can be used for rigid for elastic rotor analysis.

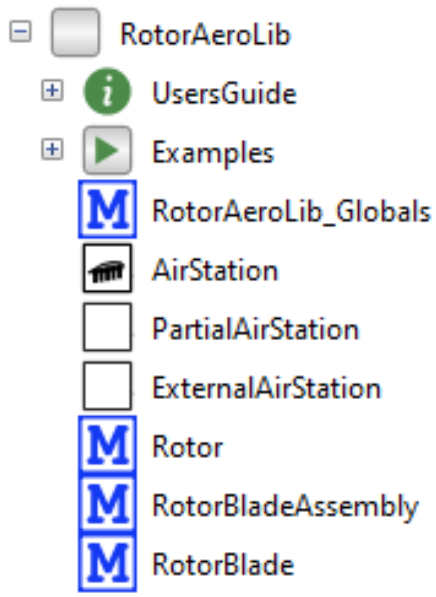

Figure 1. Top-level view of the rotorcraft aerodynamics library.

\subsection{AirStation}

At the top level of RotorAeroLib is a Modelica model that implements the aerodynamic equations for the rotor problem. This model, the AirStation, computes localized aerodynamic lift and drag sectional forces imposed upon a rotor blade as a function of local blade position, velocity, angle of attack, lift and drag coefficients, as well as global terms such as freestream velocity and overall rotor properties as described by blade element theory (BET) (Stepniewski and Keys, 1984).

In BET (also known as strip theory or lifting line aerodynamics), the rotor blade is assumed to act as a one-dimensional beam and is discretized along its length where the aerodynamic equations of each blade crosssection (i.e., an airstation) are solved independently considering local conditions (Leishman, 2006). The resultant forces are then summed along the blade length to derive total thrust and rotor torque.
Following the variable definitions for the airstation aerodynamics shown in Figure 2, the inflow angle $\phi$ is calculated via the perpendicular $U_{P}$ and tangential $U_{T}$ air flow speeds via

$$
\phi=\operatorname{atan}\left(\frac{U_{P}}{U_{T}}\right)
$$

which is related to the geometric/collective angle $\theta$ and ultimately the angle of attack $\alpha$ via

$$
\alpha=\theta-\phi \text {. }
$$

Lift $\Delta L$ and drag $\Delta D$ forces at an airstation can then be computed from

$$
\begin{aligned}
& \Delta L=\frac{1}{2} \rho U^{2} c C_{l} \Delta r \\
& \Delta D=\frac{1}{2} \rho U^{2} c C_{d} \Delta r
\end{aligned}
$$

where

$$
U^{2}=U_{P}^{2}+U_{T}^{2}
$$

is the total cross-sectional airstream speed (neglecting radial flow), $\rho$ is the density of air, $C$ is the blade chord length, $\Delta r$ is the lengthwise blade discretization size (i.e., the width of the airstation), and $C_{l}$ and $C_{d}$ are the airfoil coefficients of lift and drag, respectively, defined here by

$$
\begin{aligned}
& C_{l}=C_{l, 0}+C_{l, \alpha} * \alpha+C_{l, \alpha^{2}} * \alpha^{2} \\
& C_{d}=C_{d, 0}+C_{d, \alpha} * \alpha+C_{d, \alpha^{2}} * \alpha^{2} .
\end{aligned}
$$

Such analytic forms of sectional lift coefficients are typically obtained from wind tunnel measurements and have been relatively common in rotor aerodynamic analyses since the early days of aviation (Knight and Hefner, 1937). An alternative approach involves defining large tables of sectional aerodynamic data, known as C-81 tables, after the name of the computer software originally developed by Bell Helicopter (Harris, 2012). The C-81 tables typically include sectional lift, drag, and pitching moment as a function of angle of attack, Reynolds number, and Mach number and therefore are nontrivial to construct even with the large collection of experimental data for common airfoil sections available (Abbott and von Doenhoff, 1959). While these tables are undoubtedly more complete, the accessibility of the above analytic forms offer a straightforward implementation in RotorAeroLib that could, if desired, be expanded to use a lookup table.

The resulting lift $\Delta L$ and drag $\Delta D$ forces are then transformed into the local AirStation frame, which is oriented at the blade geometric angle (Figure 2), using

$$
\begin{aligned}
& \Delta F_{z}=\Delta L \cos (\alpha)+\Delta D \sin (\alpha) \\
& \Delta F_{y}=\Delta L \cos (\alpha)-\Delta D \sin (\alpha) .
\end{aligned}
$$



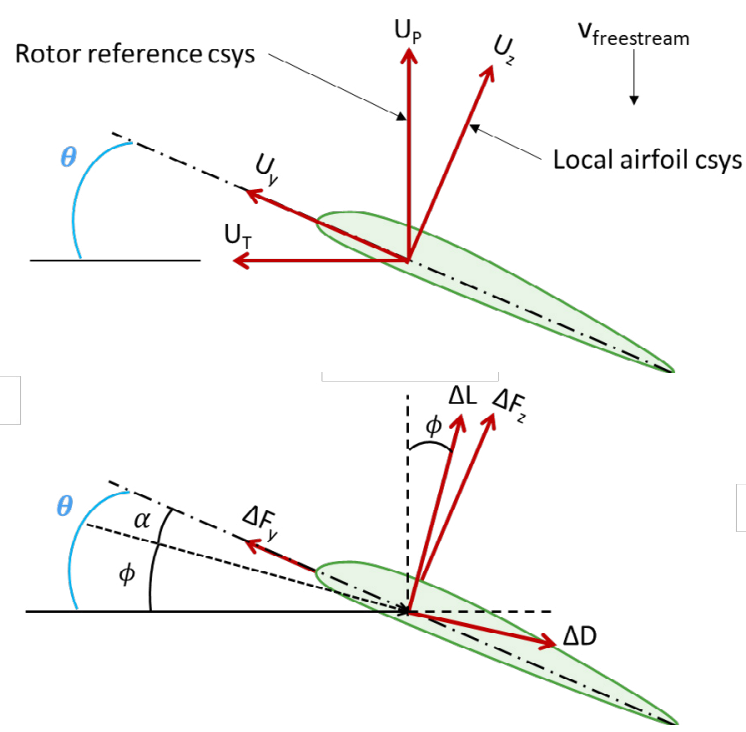

Figure 2. Variable definitions for the AirStation aerodynamics.

For the AirStation, these equations are solved in a reference coordinate system that is assumed to be oriented with $\mathrm{X}$ along the nominal blade direction and $\mathrm{Z}$ perpendicular to the nominal rotor plane. Thus, this coordinate system will rotate with the blade azimuth, but it is unaffected by flap or lead-lag blade motion. The geometric angle of an AirStation is then computed as the relative rotation about the $\mathrm{X}$-axis between the AirStation frame and the relative coordinate system. This angle continuously changes as the blade flaps, lead-lags, and feathers and is thus tightly coupled to the blade motion and aerodynamics.

As in BET, it is the intent that several AirStation models are distributed along each rotor blade in a discretized fashion so that as the number of AirStation models increases, the solution converges to the asymptotic limit. RotorAeroLib provides a discretized rotor blade model that serves this purpose, which is described in a later section.

The AirStation model extends the PartialAirStation model, which contains the basic input/output quantities necessary to provide an interface to an arbitrary aerodynamic solver. Whereas the AirStation model provides a pure Modelica implementation of the rotor aerodynamic equations, the ExternalAirStation model does the same but provides an external $\mathrm{C}$ function interface for implementing aerodynamic loading from an external source such as a computational fluid dynamics solver or one of several high-fidelity rotor aerodynamics software packages. Work utilizing ExternalAirStation is ongoing, and the model will be updated within RotorAeroLib as functional interface codes are implemented and validated.

In addition to the standard BET equations, the AirStation model can optionally include various aerodynamic correction terms to improve the accuracy of the solution. These terms include corrections for induced airflow, Mach effects, tip loss, aspect ratio, unsteady circulatory aerodynamics, and unsteady noncirculatory aerodynamics. Boolean parameters controlled by an outer RotorAeroLib_Globalsmodel (to be described next) turn these contributions on and off. Some of the computations for these corrections, e.g., unsteady noncirculatory aerodynamics, will significantly increase computational cost due to the introduction of additional states and nonlinear terms, but some flight conditions require them to ensure accuracy of the results. The following subsections describe each of the aerodynamic terms available in the AirStation model.

\subsubsection{Aerodynamic Parameter useInflowCorrection}

One of the shortfalls of BET is that it uses an assumed inflow condition. In the case of the AirStation model, the inflow is assumed to be uniform and independent of collective or blade twist, which is inaccurate for many flight conditions. An improvement for axial flight corrects the inflow using blade element momentum theory (BEMT) (Leishman, 2006) in which inflow ratio $\lambda$ and perpendicular airflow speed are corrected at each airstation via the equations

$$
\begin{aligned}
& \lambda^{2}+\left(\frac{\sigma C_{l, \alpha}}{8}-\lambda_{c}\right) \lambda-\frac{\sigma C_{l, \alpha}}{8} \theta \tilde{r}=0 \\
& U_{P}=V_{r}+\lambda V_{t i p}
\end{aligned}
$$

where $\sigma$ is the rotor solidity, $C_{l, \alpha}$ is the lift curve slope, and $\lambda_{c}$ is the inflow due to axial climb rate. With BEMT, the inflow ratio is now a function of collective and blade twist, which leads to more accurate solutions.

Although BEMT provides an improved estimate of the rotor disk inflow conditions for axial flight, the inflow is assumed to respond instantaneously to changes in rotor operating conditions. These assumptions are typically appropriate for performance prediction as well as certain stability analyses that involve high inflow conditions (e.g., propellers in forward flight). Although not implemented within the current version of RotorAeroLib, more advanced models (Leishman, 2006), such as dynamic inflow and free wake models, exist for the purpose of extending applicability to a broader problem set.

\subsubsection{Aerodynamic Parameter useAspectRatioCorrection}

The rotor aspect ratio is the ratio of the blade length to its mean chord length, defined as

$$
A_{R}=0.75 \frac{R}{c}
$$

where $R$ is the blade length (Bland and Bennett, 1963). The 0.75 factor comes from the aspect ratio at the threequarters radius. The aspect ratio correction factor $F_{A R}$ is defined as

$$
F_{A R}=\frac{A_{R}}{A_{R}+2}
$$


and accounts for tip loss (Bland and Bennett, 1963). This correction factor is applied directly to the AirStation lift value.

\subsubsection{Aerodynamic Parameter useMachCorrection}

As a blade rotates, the tangential, and thus total, air speeds it encounters are a function of radial position along its length. Full-scale vehicles commonly have tip Mach numbers in excess of 0.6 , warranting the use of the Prandtl-Glauert compressibility correction. The compressibility correction factor takes into account these effects individually at each AirStation since each will have a different Mach number (Bland and Bennett, 1963). With the speed of sound $c_{S}$, the Mach number defined as

$$
M=\frac{U}{c_{S}}
$$

feeds into the Mach correction factor $F_{M}$ as

$$
F_{M}=\frac{1}{\sqrt{1-M^{2}}}
$$

which then gets applied directly to the AirStation lift value.

\subsubsection{Aerodynamic Parameter useTipLossCorrection}

Finite lifting wings encounter a phenomenon termed "tip loss," which is caused by the high-pressure air beneath the airfoil sections rolling over the blade tip to the low-pressure side above the airfoil, resulting in a loss of lift. Accounting for these effects in rotary wing aerodynamics is naturally more challenging than fixed wing aerodynamics due to the unsteady aerodynamics. There exists numerous empirical tip loss models (Leishman, 2006; Stepniewski and Keys, 1984) to incorporate these effects into simplified aerodynamic computations. The implementation within RotorAeroLib utilizes a tip loss model similar to that used in the Dymore comprehensive solver (Bauchau, n.d.). In the Modelica model, blade section lift is modified by the correction factor

$$
F_{\text {tiploss }}=\tanh \left(\frac{1-\frac{r}{R}}{1-\mu}\right)
$$

where $r$ is the radial distance along the blade of the airstation and $\mu$ is a user-prescribed tip loss coefficient, typically selected between 0.95 and 1.0. The resulting tip loss factor is approximately unity near the rotor hub and increases significantly near the blade tip where tip losses are at their greatest. This correction factor is applied directly to the AirStation lift value.

\subsubsection{Aerodynamic Parameter useUnsteadyAero}

Rotor aerodynamics tend to be dominated by unsteady effects, especially at the low inflow flight conditions typically encountered by conventional helicopter main rotors. This is a consequence of several rotor phenomena and is largely attributed to a combination of cyclic controls (i.e., commanded blade pitch becomes a function of rotor azimuth), tip vortex trajectories (both periodic and aperiodic), and blade structural dynamics (including pitch, flap, and lead-lag motion, along with local aeroelastic deformations).

One method for incorporating unsteady aerodynamics involves application of Wagner's function. The value of the lift coefficient $C_{l}$ at angle of attack $\alpha$ can be represented by a state space system approximation of the Wagner function (Leishman and Nguyen, 1990) as

$$
\begin{gathered}
{\left[\begin{array}{l}
\dot{x}_{1} \\
\dot{x}_{2}
\end{array}\right]=\left[\begin{array}{cc}
0 & 1 \\
-0.01375\left(\frac{2 U}{c}\right)^{2} & -0.3455\left(\frac{2 U}{c}\right)
\end{array}\right]\left\{\begin{array}{l}
x_{1} \\
x_{2}
\end{array}\right\}+} \\
\left\{\begin{array}{l}
0 \\
1
\end{array}\right\} \alpha(t)
\end{gathered}
$$

$$
\begin{gathered}
C_{l}(t)=C_{l, \alpha}\left[0.006825\left(\frac{2 U}{c}\right)^{2} \quad 0.10805\left(\frac{2 U}{c}\right)\right]\left\{\begin{array}{l}
x_{1} \\
x_{2}
\end{array}\right\}+ \\
\frac{C_{l, \alpha}}{4} \alpha(t)
\end{gathered}
$$

where $C_{l, \alpha}$ is the lift curve slope and $x_{1}, x_{2}$ are state variables. If selected, this set of equations fully replaces the linear equation for the lift coefficient (eq. 6).

\subsubsection{Aerodynamic Parameter useUnsteadyNoncircAero}

Unsteady noncirculatory terms (also termed "apparent mass" terms) are a result of unsteady blade accelerations. The unsteady blade motion requires a certain mass of air be moved to accommodate the blade's physical position. The acceleration of this mass of air gives rise to the noncirculatory terms.

This introduces a correction $C_{l, n c}$ to the AirStation lift coefficient defined as

$$
C_{l, n c}=\pi b\left(\frac{\dot{\theta}}{U}+\frac{\ddot{h}}{U^{2}}-\frac{b a \ddot{\theta}}{U^{2}}\right)
$$

where $b$ is the semi-chord, $\theta$ is the geometric angle, $h$ is the vertical plunge distance from the rotor plane, and $a$ is distance from the center of blade rotation to the mid-chord (Hodges and Pierce, 2002). This correction term is added to the lift coefficient from unsteady aerodynamics and is only used if the useUnsteadyAero parameter is true.

\subsection{RotorAeroLib_Globals}

The RotorAeroLib_Globals model uses a Modelica outer declaration to define a globally scoped class containing definitions of whole-rotor design parameters, variables, and the aerodynamic correction term usage flags. One RotorAeroLib_Globals model is intended to be used with each rotor, so in multi-rotor configurations, the user must take care to properly encapsulate the model within an instance of a rotor to ensure a one-toone correspondence between a rotor's RotorAeroLib_Globals model and its set of AirStation models.

Within the RotorAeroLib_Globals model are parameters for the rotor blade radius, root cutout radius, 
chord length, solidity, and tip loss factor. Also included are the air density, speed of sound, and free-stream velocity. To calculate certain aerodynamic values, it is necessary to know the nominal blade tip speed, which cannot be calculated at any given AirStation due to its purely local definition. Therefore, the RotorAeroLib_Globals model contains an input port for the rotor shaft speed from which the nominal blade tip speed is calculated. This value is then used in an AirStation model which contains an inner RotorAeroLib_Globals model.

Also defined within the RotorAeroLib_Globals model are the parameter definitions for the various AirStation correction terms. Each of these is turned on and off via a Boolean parameter setting, which then gets referenced within the AirStation model.

\subsection{RotorBlade}

A rotor blade typically has properties that vary along its length, including cross-sectional and material properties which are usually defined in terms of EI, GJ constants. Typically, there is also a cutout region near the blade root where there is no airfoil, beyond which the blade twists along its axis to account for variation in aerodynamics due to changes in transverse speed as a function of radius. The RotorBlade model provides a template with which to include these effects as parameters.

The RotorBlade model consists of a series of flexible beam members from the DeployStructLib library attached end to end with a discretization level set by a parameter. Between each beam segment is a FixedRotation model that progressively implements the twist of the beam along its length. The twist rate is set by a parameter. AirStation models are attached to a frame located at the quarter chord of each beam, rotating with the beam as it moves and imparting aerodynamic forces onto the beam. The AirStation is also connected to a reference coordinate system, which is always oriented with the blade nominal position within the rotor plane to provide a reference for the computation of the aerodynamic equations.

\subsection{RotorBladeAssembly}

A rotor can have any number of blades, so the RotorBladeAssembly model is parameterized to create multiple instances of a RotorBlade model and automatically set up the appropriate relative and reference orientations for the blades and their inputs. Most parameters are passed through this model and applied to the underlying RotorBlade models.

The RotorBladeAssembly model provided in this library uses a configuration where the blades are fixed together in the same plane, which would be representative of fixed rotor or gimballed rotor topologies. Other rotor topologies can be created by modifying this model and incorporating the appropriate rotor mechanisms (e.g., pitch links, pitch horns, and joints). An example of a gimballed rotor topology is provided in RotorAeroLib.Examples. GimballedRotor.

\subsection{Rotor}

The Rotor model is a template for properly combining a BladeAssembly and a RotorAeroLib_Globals model to ensure that the reference coordinate system is correctly set up for the RotorBlade models and is isolated from any motion due to the rotor topology mechanisms. This is important because the aerodynamic equations require a reference coordinate system fixed in the nominal blade orientation. With the exception of a fixed rotor, the flapping and lead-lag motion of the blades are perturbations upon this nominal blade orientation, so it is important that the reference coordinate system is properly isolated at a point that is outside of such motion.

The Rotor model also contains an implementation of the rotor motor or more simply the shaft speed control, which notionally could be of any type and complexity. In some cases, it is sufficient to set the rotor speed to a set value without concern about coupled motor dynamics, as is the case with the Rotor model. With the advent of electric aircraft propulsion and multi-rotor hobby or maintenance copters, electric motors are of increasing interest. An example of an electric motor coupled into the rotor drive train is provided in RotorAeroLib.Examples.ElectricRotor.

\section{Examples}

We now present three examples of rotor analysis using the RotorAeroLib to perform different types of rotorcraft analyses. The first example validates the basic BEMT implementation of the aerodynamic equations. Presented in the second example is an evaluation of the rotor whirl-flutter stability boundary and comparison to theory and test data. The final example demonstrates the altitude control of a quadcopter model, including the modeling of electric motors.

\subsection{Aerodynamics Validation}

The Modelica implementation of the aerodynamic equations in RotorAeroLib was exercised to both verify and validate the strip theory aerodynamics by replicating the Knight and Hefner five-bladed 1937 rotor experiment (Knight and Hefner, 1937). The results were also compared to an analytic model of the BEMT equations (Reveles et al., 2019). The model was discretized with eleven airstations on each rigid blade and assumed to have no tip loss. Unsteady aerodynamic terms were also turned off. The analytic model utilized a tip loss model with 100 airstations per blade. Figure 3 shows that the Modelica model has excellent agreement with both the analytic method and the test data for thrust, while Figure 4 displays the same trends for rotor power. The use of a tip loss model on the analytic curve is 
observed to slightly reduce thrust and nominally increase the predicted power required, both of which are in the direction of the experimental measurements.

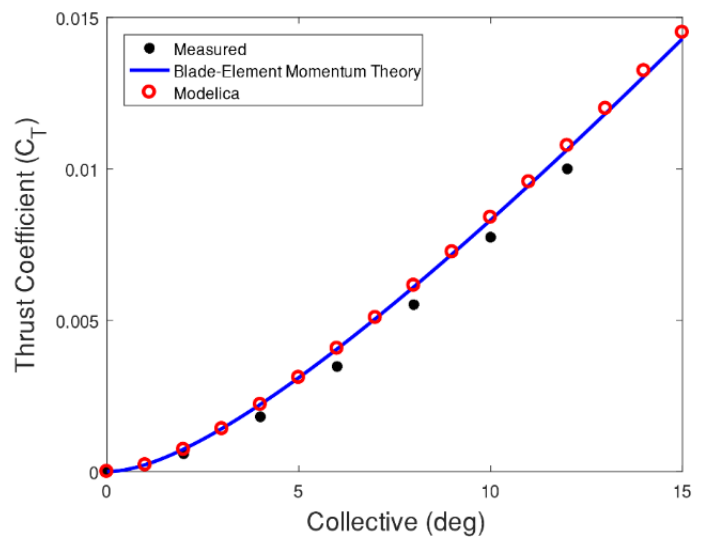

Figure 3. Thrust coefficient as a function of collective for five-bladed Knight and Hefner experiment.

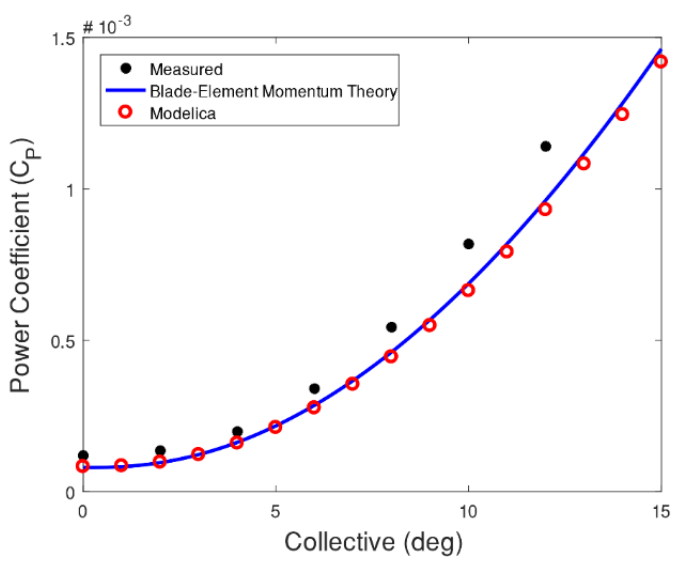

Figure 4. Power coefficient as a function of collective for five-bladed Knight and Hefner experiment.

\subsection{Whirl Flutter}

To test the ability of using the RotorAeroLib to accurately model the physics of whirl flutter, a model of the Bland and Bennett whirl-flutter experiment (Bland and Bennett, 1963) was developed. The model, shown in Figure 5, consists of a fixed rotor connected to a pylon that has pitch and yaw about its base with characteristic stiffness and damping, which represents the rotor's connection to an aircraft wing. In this model, the rotor is free to rotate and is driven only by the aerodynamics induce by the freestream air velocity and not by a motor nor by any other power source. The rotor was created with six airstations per blade, which was observed to have little difference (outside of computational speed) from a twenty-airstations-per-blade model for this problem. Built-in twist was linearized and a sectional lift coefficient of 5.7/rad was utilized without any in-plane drag coefficients. A visualization of this configuration is shown in Figure 6. The propeller was trimmed to a windmilling state (zero torque) and results compared with analytic theory. Figure 7 shows a comparison of advance ratio results, revealing good correlation between the model, analytic theory, and measurements taken from reference (Bland and Bennett, 1963).

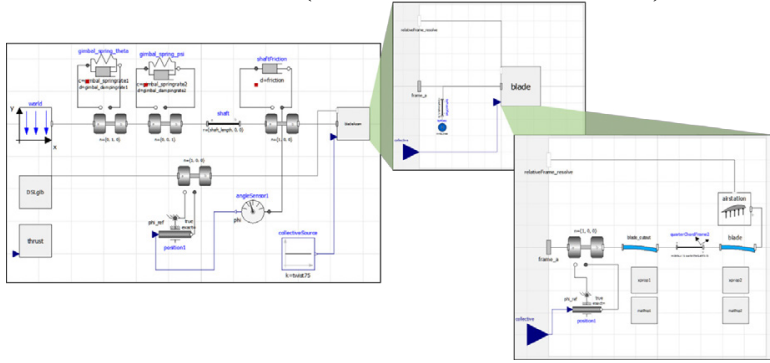

Figure 5. Block diagram for the Modelica-based whirl flutter model.

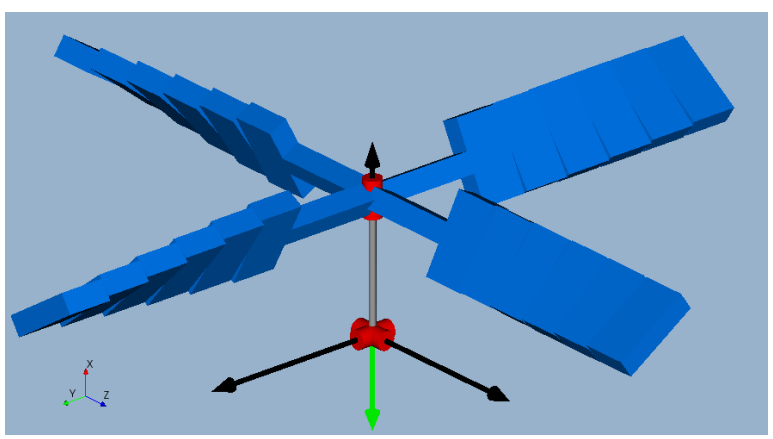

Figure 6. Modelica model of the Bland and Bennett propeller.

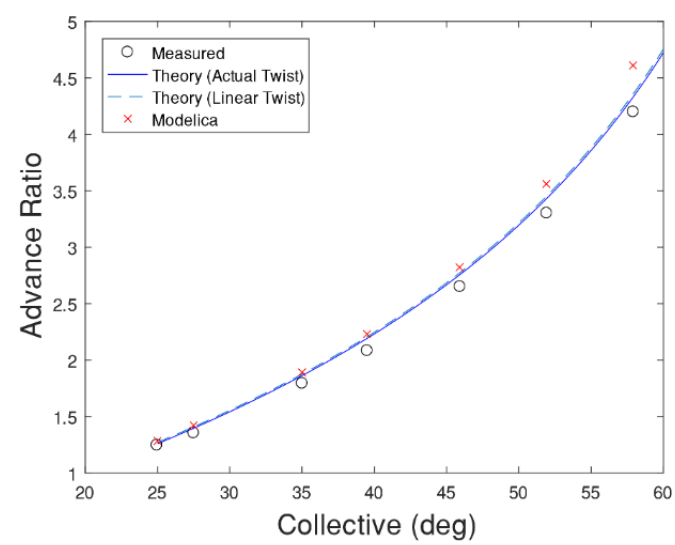

Figure 7. Advance ratio for the propeller windmilling state as a function of collective.

To solve the whirl-flutter stability problem, the Modelica model is initialized into a windmilling state (steady-state and zero torque) at which point a linearization is performed to obtain a state-space representation of the system. From this, the eigenvalues of the matrix $\boldsymbol{A}$ relating system states and their derivatives are retrieved, yielding the aerodynamic damping and frequency as the real and imaginary parts of the eigenvalues, respectively. A negative real part corresponds to a stable system, whereas a positive real 

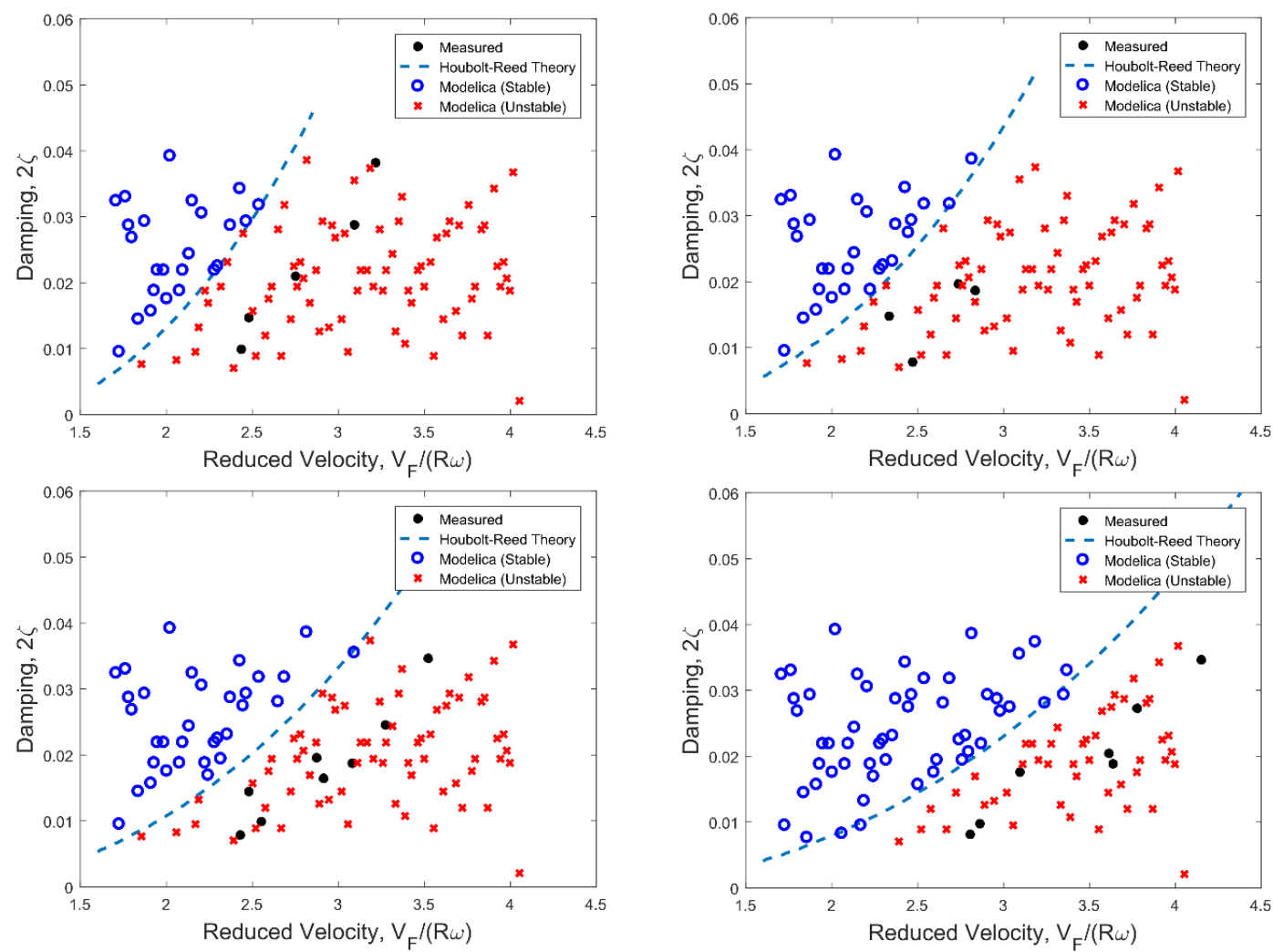

Figure 8. Comparison of whirl-flutter boundary between Modelica (blue circles are stable, red X's are unstable) and the present Houbolt-Reed analysis with only quasi-steady aerodynamic terms. Blade angles are $25^{\circ}$ (top left), $35^{\circ}$ (top right), $46^{\circ}$ (bottom left), and $58^{\circ}$ (bottom right).

part corresponds to an unstable system. By sampling the parameter space of gimbal damping rates and freestream velocity, the whirl-flutter stability boundary can be characterized by the sign of the eigenvalue real part.

The Modelica model was used to predict the whirlflutter stability boundaries using quasi-steady aerodynamics for four different collective angles: $25^{\circ}$, $35^{\circ}, 46^{\circ}$, and $58^{\circ}$. Figure 8 plots the stable and unstable test points from a parametric sweep of the operating envelope along with the Houbolt-Reed analytical model (Reveles et al., 2019) and experimental data from reference (Bland and Bennett, 1963). There exists excellent agreement between the two analysis methods, where all Modelica results align with the Houbolt-Reed stability boundaries for $25^{\circ}$ and $35^{\circ}$ collectives, and only subtle deviances are observed at the higher collective angles of $46^{\circ}$ and $58^{\circ}$. The small deviations at higher collectives are attributed to weak nonlinearities that are automatically resolved in the Modelica model. It is observed that the Modelica model results at higher collectives subtly shift the results closer to the observed measurements as compared to the Houbolt-Reed results, although without the use of unsteady aerodynamics, an accurate reconstruction of the stability boundary is not expected. It is also evident that the analytical methods show better correlation at higher collectives than at lower collectives. This may be due to the high inflow velocities encountered in the windmilling state that induce large local angles of attack, beyond which small angle assumptions may not necessarily apply. Additional deficiencies in thin airfoil theory are likely exacerbated by the relatively lower Reynolds numbers encountered at model scale that tend to induce earlier separation than those encountered at full scale.

\subsection{Quadcopter Flight Control}

The final example demonstrates the use of the RotorAeroLib on the flight control of a quadcopter model. Each rotor of the aircraft consists of a fixed rotor rigidly attached to a spar connected to the quadcopter body, which is represented by a lumped mass. For this simplified example, all four rotors rotate in the same plane and operate in a quiescent environment with zero freestream velocity. As is typical of these vehicles, two rotors rotate clockwise, while the other two rotate counterclockwise to provide a means to maintain rotational inertia balance. A control system simultaneously controls the input voltage to four rotor electric motors that drive the rotors; hence, the rotor speed is the same for all four rotors. The control system is used to set the altitude of the quadcopter and consists of a PID controller with maximum and minimum motor 
voltage limits. Figure 9 shows a visual representation of the quadcopter.

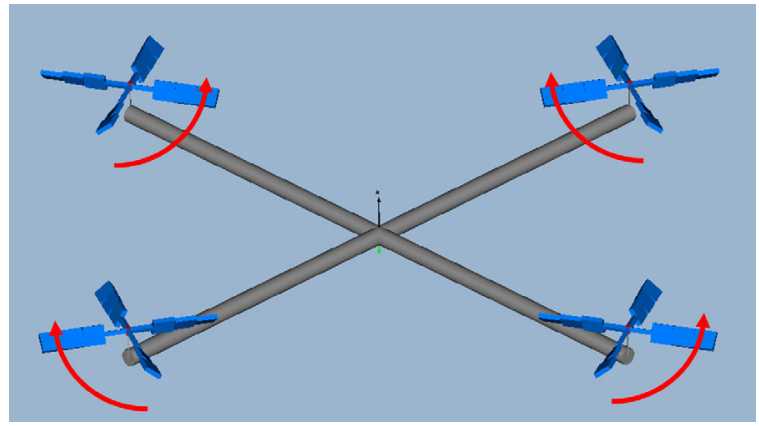

Figure 9. Visualization of the quadcopter model with rotor rotation directions identified.

A demonstration of the quadcopter model is performed by changing the altitude set point of the controller to $5 \mathrm{~m}$ after two seconds of hover at $0 \mathrm{~m}$, which is also its initialization state. Figure 10 plots the set point and quadcopter altitude, showing a small overshoot in altitude and then settling to the set point. Figure 11 shows the motor voltage input and rotor speed as the maneuver takes place, from which the coupled electro-aero-mechanical dynamics and controller voltage limits are apparent. Figure 12 plots the motor current, which further shows the electro-mechanical coupling of the system. The sizing of the rotors, motors, and other aspects of this demonstration model are somewhat arbitrary, which is clear in the very high motor current peaks, although the steady-state current is approximately $1.1 \mathrm{Amp}$.

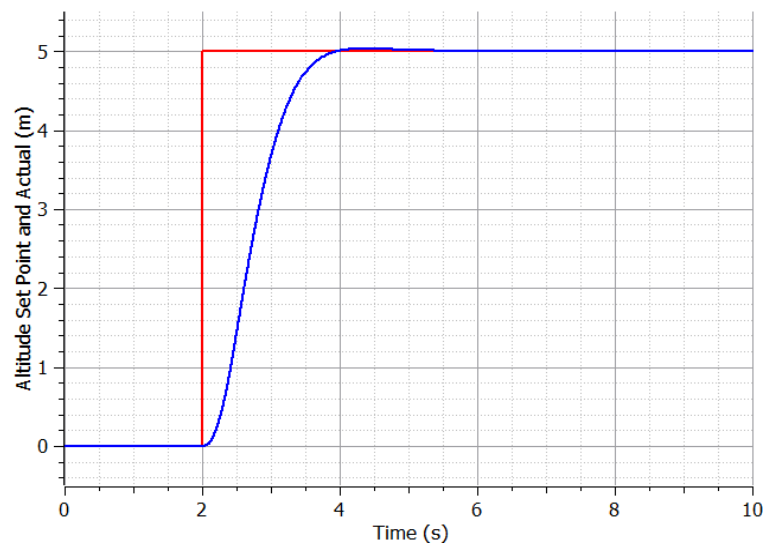

Figure 10. Quadcopter altitude set point (red) and actual (blue).

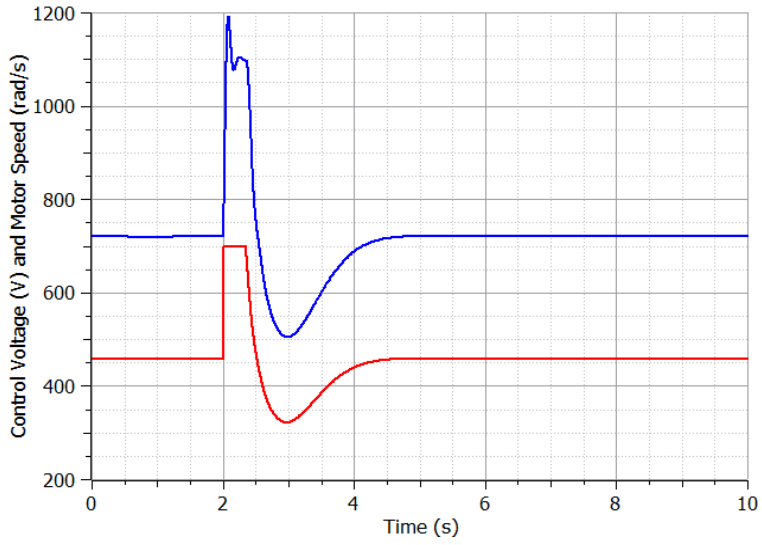

Figure 11. Quadcopter motor voltage (red) and rotor speed (blue).

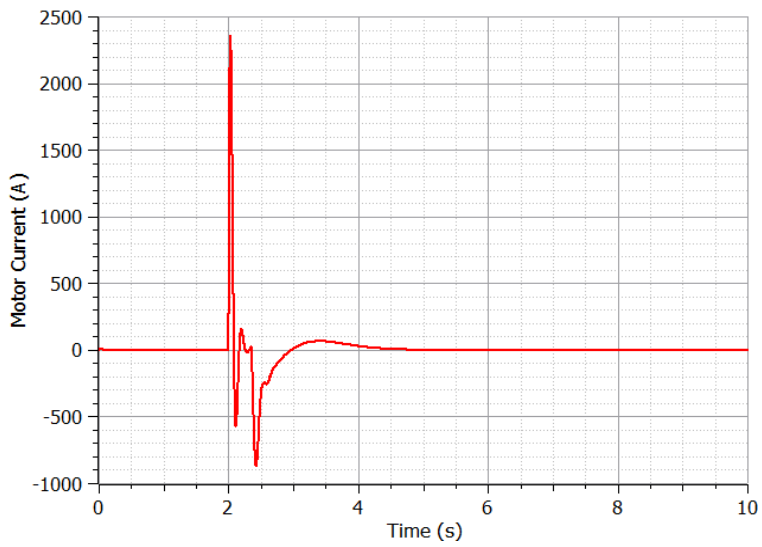

Figure 12. Quadcopter motor current.

\section{Conclusions}

This paper presents a Modelica library, RotorAeroLib, for the analysis of rotorcraft aerodynamics, whirl flutter, and rotorcraft control. It provides models useful for simulating the coupled motion of a rotor, the rotor blades, and the aerodynamic forces imparted upon the blades as they move through a fluid. The library may also be used for performing whirl-flutter analysis of rotors connected to flexible structures where dynamic instabilities may manifest.

The Rotorcraft Aerodynamics Library is open source and available via the GitHub page https://github.com/ata-engineering/RotorAeroLib which will likely be linked to via the Modelica Association website at https://modelica.org/libraries. While the library should work for any Modelica implementation per the Modelica standard, it was developed using OpenModelica and has not been tested using other software. External contributions and bug fixes or reports are encouraged.

\section{References}

I.H. Abbott and A.E. von Doenhoff. Theory of Wing Sections. Dover Publications, New York, 1959. 
J. Batteh, J. Gohl, M. Sielemann, P. Sundstrom, I. Torstensson, N. MacRae, and P. Zdunich. Development and Implementation of a Flexible Model Architecture for Hybrid-Electric Aircraft. In Proceedings of the 1st American Modelica Conference. Cambridge, MA, USA, October 2018. doi: 37 10.3384/ecp1815437.

O.A. Bauchau. Dymore User's Manual [Computer software] [Online]. University of Maryland, n.d. http://dymoresolutions.com.

S.R. Bland and R. M. Bennett. Wind-Tunnel measurement of Propeller Whirl-Flutter Speeds and Static-Stability Derivatives and Comparison with Theory. NASA TN D1807, 1963.

F.D. Harris. Introduction to Autogyros, Helicopters, and Other V/STOL Aircraft. Volume II: Helicopters. Ames Research Center, Moffett Field, CA, 2012.

D.H. Hodges and G.A. Pierce. Introduction to Structural Dynamics and Aeroelasticity. Cambridge University Press, New York, NY, 2002.

J.C. Houbolt and W.H. Reed. Propeller-Nacelle Whirl Flutter. Journal of the Aerospace Sciences, Vol. 29, No. 3, March, 1962.

M. Knight and R.A. Hefner. Static Thrust Analysis of the Lifting Airscrew. NACA TN No. 626, December, 1937.

J.G. Leishman. Principles of Helicopter Aerodynamics, 2ndedition. Cambridge University Press, New York, NY, 2006.

J.G. Leishman and K.Q. Nguyen. State-Space Representation of Unsteady Airfoil Behavior. AIAA Journal, Vol. 28, No. 5, May 1990, pp. 836-844.

W.H. Reed. Review of Propeller-Rotor Whirl Flutter. NASA TR R-264, 1967.

W.H. Reed and S. R. Bland. An Analytic Treatment of Aircraft Propeller Precession Instability. NASA TN D-659, January 1961.

N. Reveles, J. Schoneman, C. Rupp, and E. Blades. Utilizing Analytic and Direct Methods for the Verification and Validation of Whirl Flutter Analyses. In Proceedings of the AIAA SciTech Forum. San Diego, CA, USA, January 2019. doi: 10.2514/6.2019-1865.

C. Rupp and L. Schweizer. The Deployable Structures Library. In Proceedings of the 1st American Modelica Conference. Cambridge, MA, USA, October 2018. doi: 10.3384/ecp18154187.

W.Z. Stepniewski and C.N. Keys. Rotary-Wing Aerodynamics. Dover Publications, New York, 1984. 